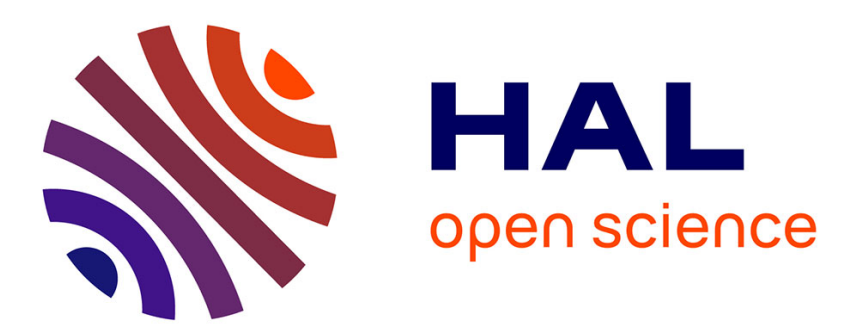

\title{
Appareil d'évaluation in vivo des propriétés rhéologiques du revêtement cutané par technique ultrasonore
}

S. Diridollou, M. Berson, D. Black, Y. Gall, L. Vaillant, F. Patat, L. Pourcelot

\section{To cite this version:}

S. Diridollou, M. Berson, D. Black, Y. Gall, L. Vaillant, et al.. Appareil d'évaluation in vivo des propriétés rhéologiques du revêtement cutané par technique ultrasonore. Journal de Physique IV Proceedings, 1994, 04 (C5), pp.C5-1251-C5-1254. 10.1051/jp4:19945277 . jpa-00252968

\section{HAL Id: jpa-00252968 https://hal.science/jpa-00252968}

Submitted on 1 Jan 1994

HAL is a multi-disciplinary open access archive for the deposit and dissemination of scientific research documents, whether they are published or not. The documents may come from teaching and research institutions in France or abroad, or from public or private research centers.
L'archive ouverte pluridisciplinaire HAL, est destinée au dépôt et à la diffusion de documents scientifiques de niveau recherche, publiés ou non, émanant des établissements d'enseignement et de recherche français ou étrangers, des laboratoires publics ou privés. 


\title{
Appareil d'évaluation in vivo des propriétés rhéologiques du revêtement cutané par technique ultrasonore
}

\author{
S. DIRIDOLLOU ${ }^{* *}$, M. BERSON, D. BLACK ${ }^{* *}$, Y. GALL ${ }^{* *}$, L. VAILLANT ${ }^{*}$, F. PATAT et \\ L. POURCELOT
}

INSERM U316 et GIP Ultrasons de Tours, Faculté de Médecine, 2 bis boulevard Tonnellé, 37032 Tours cedex, France

* Service de Dermatologie, Hôpital Trousseau, 37044 Tours, France

${ }^{* *}$ Centre Jean Louis Alibert, CHU Rangueil, Bâtiment L1, 31054 Toulouse, France

résumé: In certain dermatoses, the mechanical properties of the skin are markedly altered. The evaluation of these properties in-vivo requires equipment which can simultaneously measure the skin's thickness while applying a known stress to the site in question. Skin thickness can be measured with either A- or B-scan mode ultrasound, the former of which we have incorporated in a device with enables skin elasticity to be determinated. Essentially, this involves the measurement of the distance between transducer $(20 \mathrm{Mhz})$ and the skin structures, before and after deformation by suction, with a resolution of $70 \mu \mathrm{m}$. The transducer is located inside a small, cylindrical chamber, which has a $6 \mathrm{~mm}$ diameter aperture below the transducer to allow contact with the skin. This device can determine, in a non-invasive way, not only which skin structures are involved in this deformation, but also the morphological variation in the subcutaneous layers and their level of involvement at different stresses. With such information, we hope to be able to propose a mechanical model and to define appropriate skin rheological parameters.

\section{INTRODUCTION.}

L'étude des propriétés mécaniques de la peau dans des situations physiologiques et pathologiques, permet de qualifier et de quantifier certaines altérations dermiques, et d'évaluer l'efficacité de leur thérapeutique. Elle peut aussi permettre de mieux cerner les effets des produits cosmétiques sur la peau (hydratation, souplesse..), vis à vis des agressions extérieures et du vieillissement cutané (actinite ou intrinsèque) $[1][2]$.

L'étude des propriétés mécaniques d'un matériau est réalisée en appliquant un champ de contrainte sur celui-ci et en mesurant le champ de déformation résultant. De part la complexité du revêtement cutané, son étude mécanique pour être précise et complète requiert la mesure des déformations de ses différentes structures, et celle de l'élément de volume sollicité par la contrainte [3]. Malheureusement les appareils existants ne mesurent que le déplacement de la surface cutanée soumis à une contrainte, ce qui ne suffit pas à caractériser mécaniquement la peau.

Dans cet article nous présentons un appareil non invasif qui permet cette caractérisation. Les progrès récents de l'échographie haute résolution de la peau ont permis de visualiser de manière non invasive en temps réel et avec une grande précision les structures cutanées [4]. C'est pourquoi nous avons 
développé un appareil utilisant une technique ultrasonore qui permet d'avoir une meilleure connaissance du champ de déformation en profondeur.

\section{MATERIEL.}

L'appareil mis au point au laboratoire est appelé échorhéomètre. Il est composé: d'un dispositif de succion qui crée par l'intermédiaire d'une cuve posée sur la peau un champ de contrainte sur celle-ci, d'une technique ultrasonore qui permet l'investigation des différentes structures cutanées au cours de la déformation, et d'un micro-ordinateur qui gère d'une part l'ensemble du dispositif, et d'autre part la visualisation et l'exploitation des résultats.

Plus précisément l'expérience se déroule comme suit: une petite cuve appelée sonde de dépression (figure 1), est posée sur la peau. La dépression est créée dans la cuve par l'intermédiaire de l'orifice de dépression. L'ouverture de la cuve de diamètre $\mathrm{L}_{0}(6 \mathrm{~mm})$ délimite la surface de peau sollicitée par la contrainte, et permet le passage du faisceau ultrasonore. La propagation des ultrasons se fait dans une solution aqueuse en raison de l'atténuation très élevée à haute fréquence dans l'air.

Le transducteur ultrasonore est fixé à l'extrémité d'un axe central inséré dans la cuve. Le transducteur fonctionne en mode pulsé à une fréquence centrale de 20 Mhz. Les caractéristiques principales de ce capteur sont une focalisation mécanique de $7 \mathrm{~mm}$, et une très large bande passante ( 20 à $25 \mathrm{MHz}$ à $-6 \mathrm{~dB}$ ) qui permet une résolution axiale de $70 \mu \mathrm{m}$.

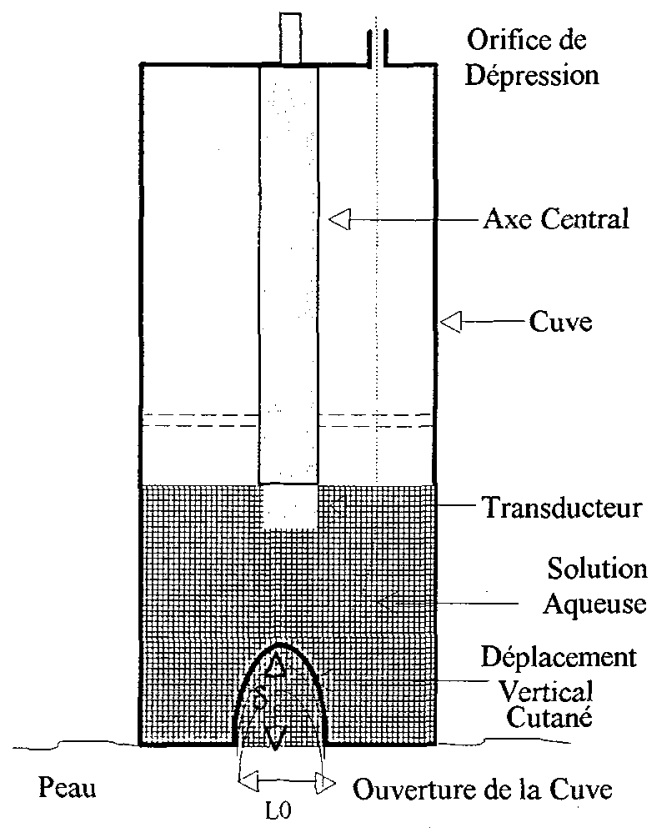

Figure1 :

\section{RESULTATS}

\subsection{Détection automatique du premier écho}

L'appareil détecte automatiquement le premier écho reçu traduisant l'interface entre la solution aqueuse et la surface de la peau. Les mesures du déplacement vertical de la surface du revêtement cutané soumis à une succion peuvent être réalisées lors de deux essais, l'écrouissage ou le fluage.

\subsection{1 essai d'écrouissage}

La dépression imposée dans la cuve est tout d'abord linéaire continue croissante telle que $p(t)=$ $\lambda t$, puis décroissante avec cette fois-ci un horaire $p(t)=-\lambda t$. Les temps de montée et de descente peuvent être choisis, ainsi que la dépression maximale et le nombre de cycles (montée et descente). La tolérance de la dépression maximale et la linéarité sont inférieures à $5 \%$ (figure 2 ). La pression relative appliquée à la peau est représentée en fonction du déplacement vertical de celle-ci, soit (f(déplacement) $=$ Pression) (figure 3 ).

Comme le point représentatif ne décrit pas au retour la même courbe qu'à l'aller, un phénomène d'hystérèse mécanique est observé, qui caractérise le comportement viscoélastique de la peau. 


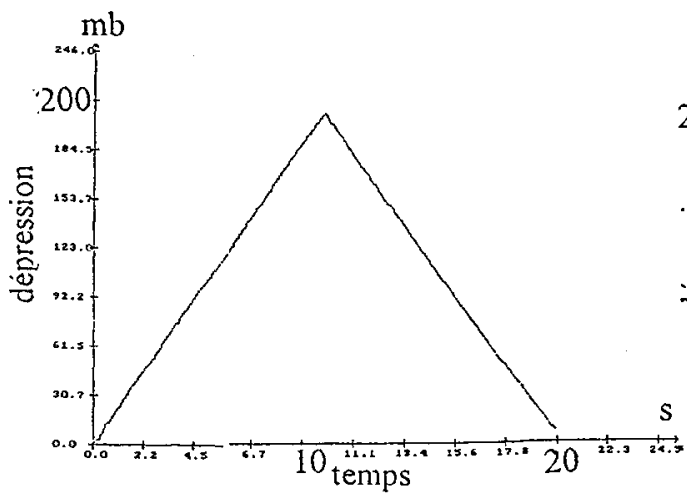

Figure 2:

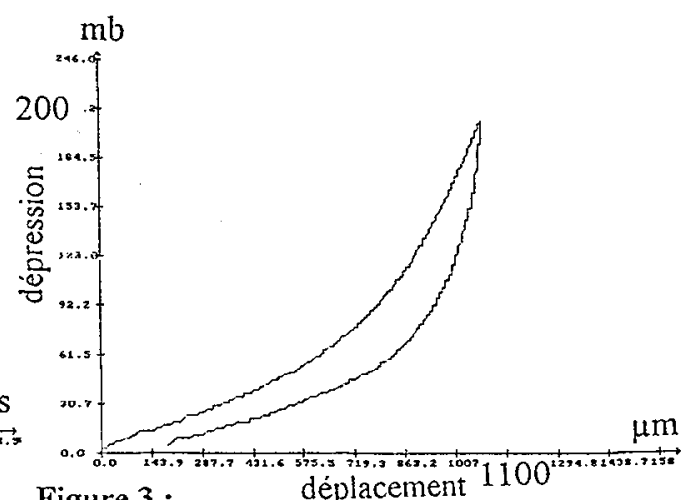

Figure 3 :

La figure 2 présente la mesure de la dépression (mbar) exercée sur la peau en fonction du temps(s), pour une pression maximum de $-200 \mathrm{mb}$ et pour des temps de $10 \mathrm{~s}$ en montée et en descente. La figure 3 présente la relation entre le déplacement vertical de la surface cutanée ( $\mu \mathrm{m})$ et la dépression exercée(mbar).

\subsection{2 essai de fluage}

Lors de cette expérience un ou plusieurs échelons de dépression sont imposés cette fois-ci à la peau. La dépression nulle depuis un temps suffisamment long (la peau au repos) est brusquement portée à une valeur constante $\mathrm{P}_{0}$, puis supprimée tout aussi rapidement (figure 4). Le temps de maintient à dépression voulue ou à pression atmosphérique, et le nombre de cycles peuvent être choisis séparément. Les temps d'application et de retrait de la dépression dans la cuve sont respectivement inférieurs à $80 \mathrm{~ms}$ et $50 \mathrm{~ms}$. La tolérance de la dépression est de $5 \%$. Le déplacement vertical de la surface cutanée est représenté en fonction du temps, soit $\delta=f(t)$ (figure 5 ). Un phénomène de fluage est observé d'où le nom de l'expérience.
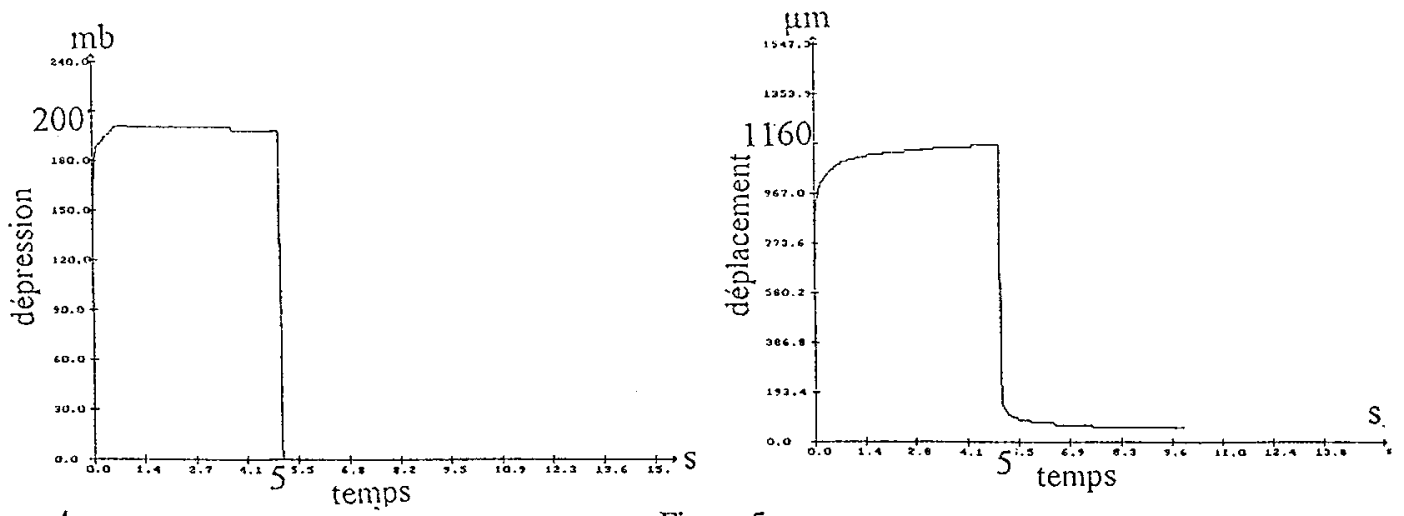

Figure 4:

Figure 5:

La figure 4 présente la mesure de la dépression (mbar) exercée sur la peau en fonction du temps(s), la dépression est de $-200 \mathrm{mb}$ pour des temps de 5 s en charge et en décharge. La figure 5 présente la relation entre le déplacement vertical de la surface cutanée $(\mu m)$ et le temps(s).

\subsection{Visualisation de la défornation des structures cutanées}

Cet appareil permet aussi d'étudier les déformations en profondeur de la peau, et de déterminer les éléments de volume sollicités. En effet la technique ultrasonore haute résolution permet la récupération des échos des différentes structures cutanées (figure 6,7), la visualisation des interfaces: solution aqueuseépiderme (a) et derme-hypoderme (b), et la mesure des épaisseurs: derme-épiderme (c) et hypoderme (d), avant et pendant l'application d'une dépression . 

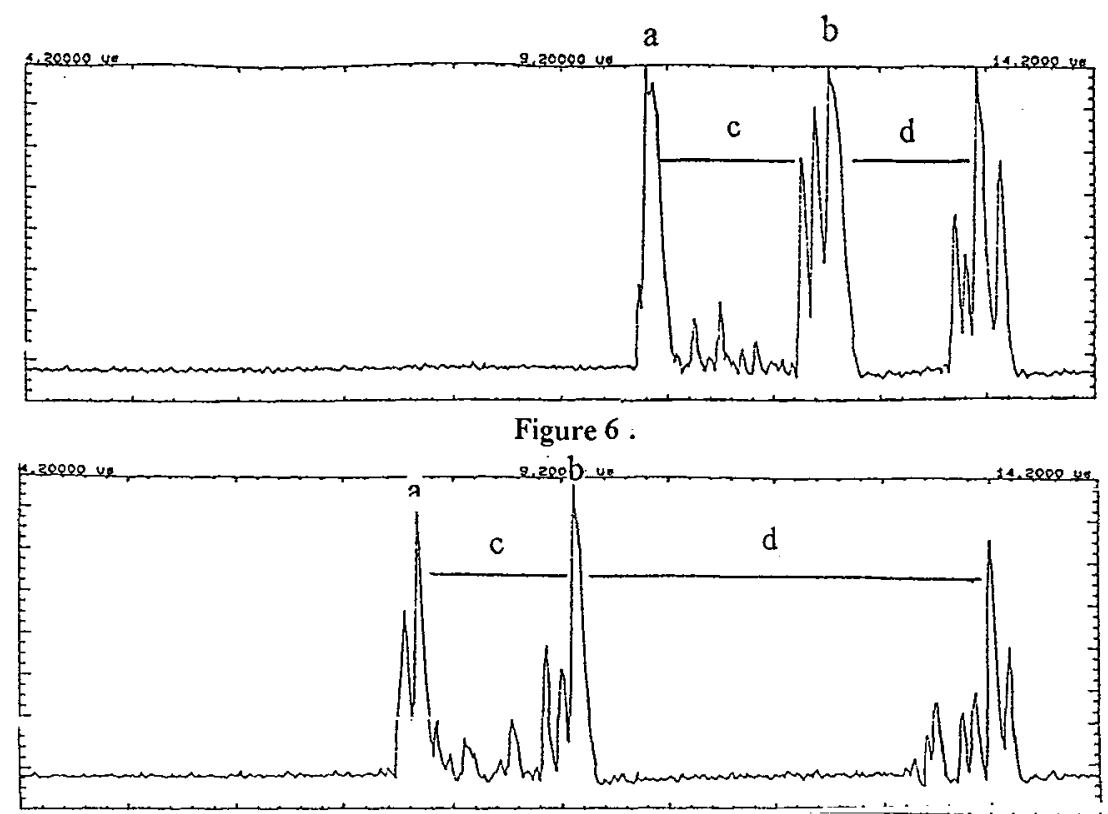

Figure 7 :

A pression atmosphérique (figure 6), l'épaisseur "derme+épiderme" est de $1.6 \mathrm{~mm}$, pour une épaisseur de l'hypoderme de $1.2 \mathrm{~mm}$. Pour une dépression relative de -300 mbar (figure 7), l'épaisseur "derme + épiderme" est de $1.4 \mathrm{~mm}$, pour une épaisseur de l'hypoderme de $3.1 \mathrm{~mm}$. Amplitude $1 \mathrm{v} /$ division, $c=1600 \mathrm{~m} / \mathrm{s}$, base de temps les/division.

\section{CONCLUSION.}

L'échorhéomètre est un outil de mesure original et performant des propriétés rhéologiques cutanées. Il permet de manière non invasive et in vivo d'exercé un champ de contrainte homogène sur le revêtement cutané, et de bien définir le champ de déformation cutanée résultant. Il détermine les structures cutanées sollicitées par la dépression, leurs variations morphologiques et leurs chronologies de recrutement. On espère maintenant avec de tels renseignements définir des paramètres rhéologiques caractérisant judicieusement les propriètés mécaniques cutanées. Ceci permettra de relier ces paramètres aux constituants structuraux de la peau, afin d'élaborer de nouveaux modéles mécaniques du tissu cutané.

Des expérimentations sur des sujets sains, sur différents sites èt classes d'âges, permettront de constituer des bases de données qui définiront la "qualité" mécanique cutanée d'un sujet, étape préliminaire avant d'aborder des travaux sur des sujets pathologiques.

\section{BIBLIOGRAPHIE}

[1] C. Escoffier Age Related Mechanical Properties of Human Skin: an in Vivo Study. The Journal of Investigative Dermatology. Vol $93 \mathrm{~N} 3$, September 1989,p-p 353-357.

[2] J. De Rigal, C. Escoffier, B. Querleux, B. Faivre, P. Agache, J.L. Lévèque. Assessement of aging of the human skin on vivo ultrasonic imaging. J. Invest. Dermatol. 1989;93:621-5

[3] T.Cook, H.Alexander, M.Cohen. Experimental Method for Determing the 2 Dimensional Mechanical Properties of Living Human Skin. Med-Biol-Eng- Comput. 1977;15:381-90.

[4] M. Berson, L. Vaillant, F. Patat, L. Pourcelot. High-Resolution Real Time Ultrasonic Scanner. Ultrasound in Medecine and Bio. 1992. Vol 18 No 5, pp 471-478. 